\title{
Down syndrome in the neurology clinic: Too much? Too little? Too late?
}

\section{Andrew J Larner}

This paper presents a review of all patients with Down syndrome seen over a 5 -year period by one consultant neurologist in general outpatient and specialist cognitive function clinics. It revealed only 7 cases in $>4500$ general referrals $(=0.2 \%)$, all referred with suspected seizure disorders. The diagnosis of epilepsy was confirmed in 6 patients. Only one new, comorbid, diagnosis was made. Neurologists have little exposure to, and hence little chance to develop expertise in, the neurological complications of Down syndrome. It is suggested that closer liaison between neurologists and psychiatrists with an interest in learning disability might improve the management of neurological problems in patients with Down syndrome.

Down syndrome, the most common chromosomal abnormality, may be associated with a variety of neurological complications including epileptic seizures, stroke (perhaps related to congenital heart disease), cervical spinal cord compression (related to atlanto-axial joint anomalies), and basal ganglia damage ${ }^{[1]}$. In addition, all patients with Down syndrome develop Alzheimer's disease-like neuropathology, sometimes associated with the clinical correlate of dementia ${ }^{[2-4]}$. For these reasons, one might anticipate that patients with Down syndrome would be encountered in neurology outpatient clinics. However, prior studies of the frequency and/or utility of neurological consultation for patients with Down syndrome have not been identified.

\section{Materials and methods}

Records of the author's general neurology outpatient clinics based in two district general hospitals (DGH) and a regional neuroscience centre, and special interest Cognitive Function Clinic based in the regional neuroscience centre, were examined for the 5-year period 2002-2006 inclusive to identify consultations with patients with a diagnosis of Down syndrome. Referral source, indication for referral, neurological diagnosis or diagnoses, and outcome were noted for all cases. No cases with unspecified causes of learning disability, nor cases with other defined chromosomal abnormalities ${ }^{[5]}$, were included.

\section{Results}

Of 4590 new patient consultations seen in general neurology outpatient clinics over the 5-year study period, there were 8 new consultations with patients with Down syndrome $(=0.2 \%)$; all were seen in DGH clinics. The number of patients with Down syndrome seen ranged from $0-3$ per year. Of 436 new patients assessed in the Cognitive Function Clinic, none had Down syndrome.

The 8 Down syndrome patient episodes (TABLE 1) involved 7 different patients (one patient was referred twice during the study period; $\mathrm{M}: \mathrm{F}=5: 2$, age range $31-52$ years, median age 46.5 years). All referrals were made by the patient's general practitioner. Three patients were already under the care of a psychiatrist with an interest in learning disability. Three patients were in residential care establishments; the others were living at home, sometimes with additional care support.

All patients were referred because of episodes of impaired consciousness which their general practitioners suspected to be due to epilepsy. Four patients were already receiving anti-epileptic drugs (AEDs) at the time of referral. Three patients already had a diagnosis of dementia, although none were receiving cholinesterase inhibitors or memantine. No patient attended alone; all came with a relative or carer or both, although in one case the carer had not witnessed any of the episodes of impaired consciousness 
and so was unable to provide any useful collateral history.

As a consequence of neurological consultation, six of the patients were judged to be suffering epileptic seizures; in the other patient loss of consciousness was thought to be due to a syncopal episode.

In three of the patients diagnosed with epilepsy, the clinical features were consistent with a syndromic diagnosis of late-onset myoclonic epilepsy in Down syndrome (LOMEDS) ${ }^{[6-7] ;}$ two of these patients already had evidence of dementia. In one of the patients with epilepsy, a concurrent diagnosis of obstructive sleep apnoea-hypopnoea syndrome (OSAHS) was suggested. This condition, which may present initially in neurology clinics $^{[8]}$, may cause or exacerbate epileptic seizures. The diagnosis was later confirmed using nocturnal pulse oximetry studies performed by a chest physician with an interest in OSAHS. This was the only new diagnosis established as a consequence of neurological consultation.

\section{Discussion}

The very small number of patients with Down syndrome identified in this 5-year review of general and specialist cognitive neurological practice prompts a number of questions, specifically, are these numbers more or less than one might expect, and are referrals occurring too late?

Few, if any, neurologists will have any specific training, far less any expertise, in the management of patients with Down syndrome, in which case any referral of a patient with Down syndrome to the neurology clinic might be deemed surprising, given the dictates of clinical governance. The numbers seen in this survey are similar to those of the most common Mendelian monogenic disorders seen in general neurology clinics (i.e. Huntington's disease, neurofibromatosis type 1$)^{[9]}$, and are likewise far too small to permit the development of any management expertise. It may be cogently argued that patients with Down syndrome are best managed in a dedicated special interest clinic.

However, in view of the possible neurological complications of Down syndrome ${ }^{[1]}$, these numbers might be deemed too small. Considering specifically the diagnosis of epilepsy, which was the

\begin{tabular}{|lllll|}
\hline Patient Sex/Age & Referral indication & On AEDs? & Dementia? & Neurological diagnosis \\
\hline${ }^{*}$ M31 & Blackouts & + & - & Epilepsy \\
${ }^{*}$ M34 & Blackouts & + & - & Epilepsy, OSAHS \\
M47 & Epilepsy & + & + & LOMEDS \\
M48 & Collapse & - & - & Syncope \\
F45 & Epilepsy & + & - & LOMEDS \\
M52 & Fitting & - & + & Epilepsy \\
F51 & Twitching & - & + & LOMEDS \\
M39 & Shouting & + & - & Epilepsy \\
*Same patient & & & & \\
Table 1 & Patient demographics, referral indications, treatment and diagnosis \\
\hline
\end{tabular}

indication for referral of all the patients seen in this survey, guidelines have previously been published suggesting a model of epilepsy care which includes subspecialty clinics to meet the needs of patients with epilepsy in learning disability ${ }^{[10]}$. Such provision may be required because insufficient neurological expertise is deemed available in dedicated learning disability clinics. The correct diagnosis of late-onset seizures in Down syndrome is crucial since this may herald onset of cognitive decline ${ }^{[11,12]}$, in the same way that seizure onset concurrent with the development of Alzheimer's disease has been documented in the non-Down syndrome population ${ }^{[13]}$, and hence be a marker for consideration of anti-dementia drug therapy. Such medications have been investigated in Down syndrome ${ }^{[4]}$, and although their exact place in management remains to be defined, it would seem likely that their greatest benefit will be in early cognitive decline. Hence it might be argued that referring patients with Down syndrome with an established diagnosis of late-onset epilepsy and already receiving anti epileptic drugs is too late, since the most appropriate time to commence anti-dementia drug therapy is already passed.

Greater communication between psychiatrists with an interest in learning disability and neurologists, perhaps even joint clinics ${ }^{[14]}$ may be required for the optimum management of neurological problems in Down syndrome. 
1. Lev N, Melamed E. Neurological complications in Down's syndrome. Harefuah. 2002; 141: 820-823.

2. Berg JM, Karlinsky H, Holland AJ, editors. Alzheimer disease, Down syndrome, and their relationship. Oxford: Oxford University Press; 1993.

3. Mann DMA. Down's syndrome and Alzheimer's disease. In: Esiri MM, Trojanowski JQ, Lee VMY, editors. The neuropathology of dementia. 2nd ed. Cambridge: Cambridge University Press; 2004. p. 207-226.

4. Prasher VP. Alzheimer's disease and dementia in Down syndrome and intellectual disabilities. Oxford: Radcliffe Press; 2005.

5. Adab N, Larner AJ. Adult-onset seizure disorder in $18 q$ deletion syndrome. Journal of Neurology. 2006; 253: 527-528.

6. Li LM, O'Donoghue MF, Sander JW. Myoclonic epilepsy of late onset in trisomy 21 . Arquivos de Neuro-Psiquiatria. 1995; 53: 792-794.
7. Möller JC, Hamer HM, Oertel WH, Rosenow F. Late-onset myoclonic epilepsy in Down's syndrome (LOMEDS). Seizure. 2001; 10: 303-306.

8. Larner AJ. Obstructive sleep apnoea syndrome presenting in a neurology outpatient clinic. International Journal of Clinical Practice. 2003; 57: 150-152.

9. Larner AJ. Monogenic Mendelian disorders in general neurological practice. International Journal of Clinical Practice. Forthcoming 2007; 61.

10. Scottish Intercollegiate Guidelines Network. Diagnosis and management of epilepsy in adults. Edinburgh: SIGN; 2003.

11. McVicker RW, Shanks OE, McClelland RJ. Prevalence and associated features of epilepsy in adults with Down's syndrome. British Journal of Psychiatry. 1994; 164: 528-532.
12. Puri BK, Ho KW, Singh I. Age of seizure onset in adults with Down's syndrome. International Journal of Clinical Practice. 2001; 55: 442-444.

13. Lozsadi DA, Larner AJ. Prevalence and causes of seizures at the time of diagnosis of probable Alzheimer's disease. Dementia and geriatric cognitive disorders. 2006; 22: 121-124.

14. National Institute for Clinical Excellence. The epilepsies: diagnosis and management of the epilepsies in adults in primary and secondary care. Clinical guideline 20. London: NICE; 2004.

Received: 08 January 2007; Accepted 14 February 2007; Published online: 30 July 2007. 